\title{
Control of Microfluidic Systems: Two Examples, Results, and Challenges
}

\author{
Micheal Armani, Satej Chaudhary, Roland Probst, Shawn Walker, and Benjamin Shapiro* \\ *Aerospace Engineering / Bio-Engineering, University of Maryland at College Park
}

(301) 405-4191, benshap@eng.umd.edu

\section{Abstract}

This paper describes results and challenges in feedback control of microfluidic systems. Results are provided for two representative examples: control of liquid droplets by electrically actuated surface tension forces and steering of many particles at once by micro flow control. Common themes and challenges are outlined based on the authors research programs and on and the results of the March 2004 NSF workshop on 'Control and System Integration of Micro- and Nano-Scale Systems’ organized by the author.

\section{Introduction}

Microfluidics refers to fluid flow inside systems whose features range in size from millimeters down to micrometers. As shown in Figure 1, this length scale is commensurate with the size of biological entities. Consequently, many microfluidic systems are aimed at applications in the bio-chemical arena. Demonstrated and under development biological applications include microarrays for rapid analysis of DNA [1], analysis and detection of proteins [2], monitoring and analysis of cells [3], and implantable drug injection systems [4].

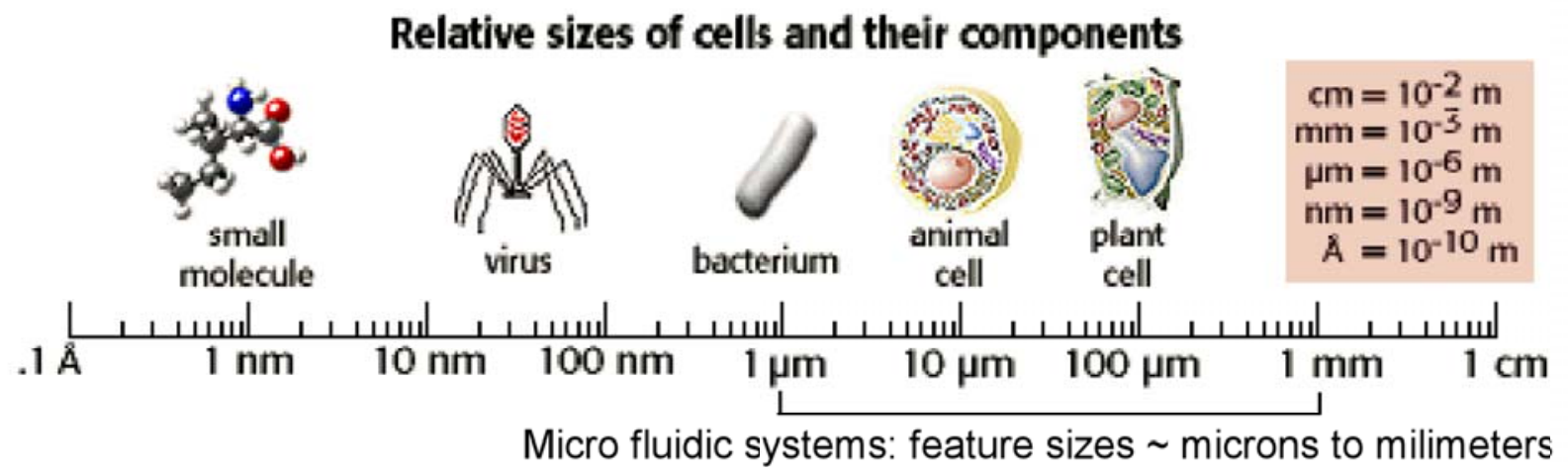

Figure 1: Device length scales compared to the size of biological entities. (Figure taken from "The Biology Project”, University of Arizona: http://www.biology.arizona.edu/cell_bio/tutorials/cells/cells2.html ).

In micro-scale applications, feedback control is needed for the same reasons that it is required on the macro-scale: micro-systems often operate in largely unknown environments and can have significant geometric, parametric, and dynamic uncertainty. Specifically, outside environments may contain unknown bio-chemical species (as in sensing applications where the presence of these species must be detected), biological fluid samples have a large degree of variability (urine samples vary with disease, hydration, and from patient to patient), and the characteristics of 
specific entities inside the samples can vary (cells of the same type will have different shapes and properties). Device geometric uncertainty can be created by fabrication limits: the wavelength of light limits fabrication techniques that use optical masks to resolutions of about a micron, hence devices with five micron sized features will have a $\pm 20 \%$ variability in geometry. Finally, models that characterize bio-chemical behavior (such as models of surface tension boundary conditions, reaction rates, diffusion, migration, species adsorption and desorption) contain uncertain parameters and unmodeled dynamics. Feedback is required to address all of these uncertainties and to create robust behavior, enable new tasks, and improve system performance.

This paper presents two representative examples of microfluidic modeling and control research in the authors laboratory: the first example deals with control of fluid packets using electrically actuated surface tension forces, the second describes individual steering of cells by micro flow control. We show how feedback control can improve performance (Sections 3) and enable new capabilities (Section 4), we outline common themes and challenges in feedback control of microfluidics (Section 5), and we briefly summarize research recommendations for control of micro/nano-scale systems in general from the March 2004 NSF workshop on 'Control and System Integration of Micro- and Nano-Scale Systems’ [5].

\section{Control of Flows Driven by Electrically Actuated Surface Tension}

Since surface to volume ratios are inversely proportional to device length scales, surface effects dominate in micro-scale systems. In particular, surface tension effects are critical in microfluidic devices [6-9]. It has been shown that it is possible to effectively modify surface tension in a variety of ways, by varying temperature [10], by modifying surface chemistry [11], by actively changing surface roughness [12], and by applying electric fields [13-15], and all of these techniques can be used to control fluid flow on the micro-scale. Figure 2 shows a liquid drop of water on top of a dielectric layer. An applied voltage between an inserted wire and an electrode underneath the dielectric layer reversibly changes the shape of the droplet.

a) no voltage

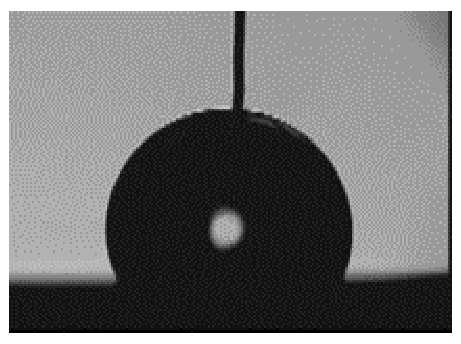

voltage $=30 \mathrm{~V}$

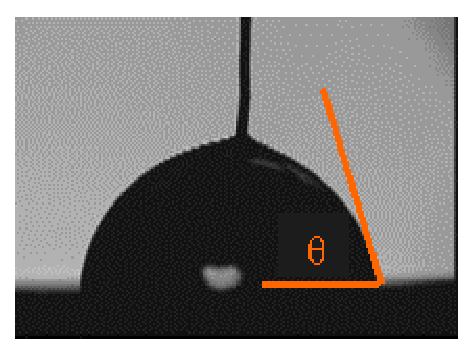

b)

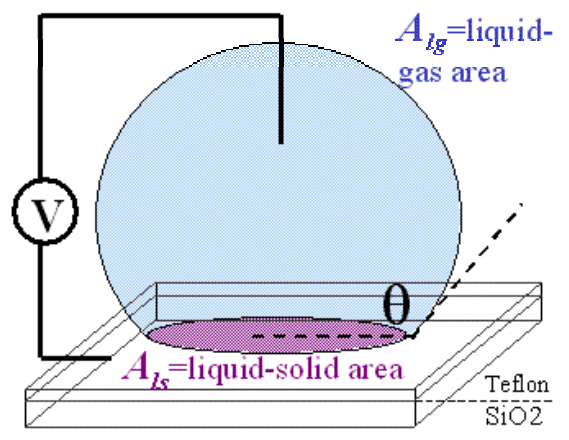

Figure 2: a) Electric-field induced change in the contact angle of a droplet of deionized, distilled water (pH 6.5) on $500 \AA$ Teflon AF coating a $1200 \AA$ thick layer of silicon dioxide. An applied voltage of $30 \mathrm{~V}$ between the inserted platinum wire and underlying gold electrode causes the droplet to flatten reversibly. (Figure courtesy of Robin Garrell at UCLA.) b) A schematic of the experiment showing the wire electrode, voltage source, hydrophobic Teflon layer, and the Silicon Dioxide dielectric (SiO2) layer. The contact angle $\theta$ and the liquid/solid interface area are also shown. 
The change in the water droplet shape is caused by a competition between electrical and surface tension energies. Teflon is a hydrophobic material and, when there is no applied voltage, the liquid water droplet beads up to minimize the area of the water/Teflon interface. When a voltage is applied, the water acts as a good conductor, and the Silicon Dioxide (SiO2) dielectric material immediately underneath the liquid/gas interface is polarized. The system electrical energy scales as this polarized volume and is at a minimum when the water/Teflon area is maximal. Thus the surface tension and electrical energies compete - as the voltage is increased the electrical contribution becomes successively stronger and the water droplet spreads [16]. This competition creates the actuation forces and determines the equilibrium shape of the liquid. Droplet dynamics depend on how the actuation forces and the loss mechanisms found at the liquid/gas interfaces (hysteresis and contact line pinning) drive the low Reynolds number, two-phase, fluid dynamics. Equilibrium and dynamic modeling results are discussed in Section 3.1.

Figure 3 shows how the competition of surface tension and electrical energies can be used to move a single liquid droplet using a ground and two active planar electrodes. The droplet will move preferentially in the direction of the applied voltage. This is the principle behind the Electro-Wetting-On-Dielectric (EWOD) lab-on-a-chip system developed by CJ Kim at UCLA [13] (also see [14, 15, 17, 18] for other examples of electro-wetting systems). The EWOD system consists of an $n$ by $m$ array of individually addressable electrodes. The system can steer multiple droplets along independent trajectories by turning on successive electrodes in the right sequence. It can split droplets by pulling on either side using three electrodes with an on/off/on voltage pattern. (It is possible to apply intermediate voltages and doing so may allow finer splitting control.) The system can transport different chemical species: either by having the chemicals mixed into water droplets or by directly actuating alternate liquids. Droplets can be merged via droplet collisions which means that the system can combine reactants and carry out chemical reactions. Finally, the system can mix materials inside droplets by forcing the droplets to execute tortuous paths that create complex flows inside the drops. (Effective mixing is a difficult issue on the micro-scale. On the macro-scale, momentum effects create vorticity and/or fluid turbulence that causes folding and interleaving of the fluid and allows diffusion to quickly complete the mixing process [19]. On the micro-scale, folding and interleaving of fluids must be created artificially: by electro-kinetic instabilities, by grooved surfaces that trip the fluid, or by mechanical moving parts [20-23].)
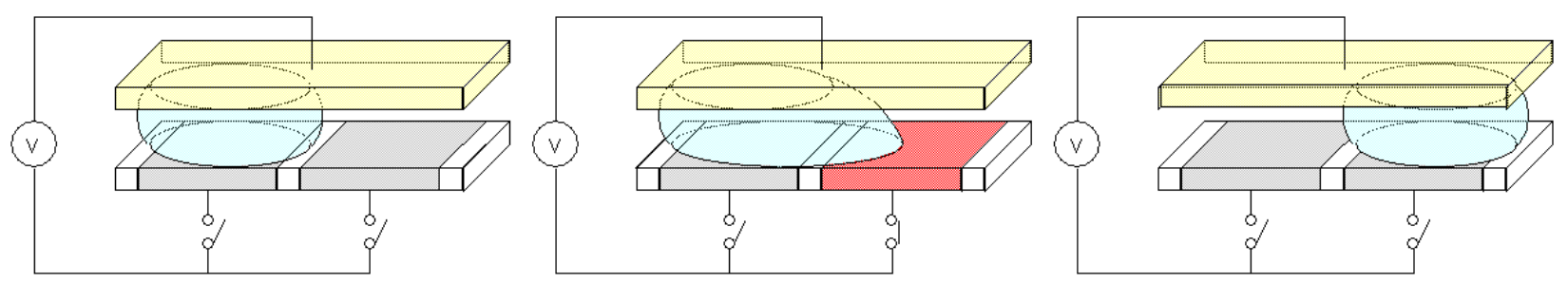

Figure 3: Illustration of fluid motion induced by an electrical potential applied across dielectric-coated electrodes above and below a liquid droplet. By switching on the voltage at an electrode adjacent to the droplet, the surface tension is effectively lowered locally causing the droplet to move to the right. (Figure courtesy Jeong-Yeal Yoon at UCLA.) 
In summary, the goal of the EWOD system is to replicate the capabilities of an entire chemical laboratory in a small hand-held device [13, 24-26]. There are many potential applications of EWOD, and other lab-on-a-chip systems [2, 27-29]. Nurses could carry hand-held devices that would screen single drops of patients blood for protein and DNA disease markers in real-time and without the necessity to send large quantities of sample back to off-site laboratories. First responders could be equipped with hand-held or remotely deployable biological and chemical pathogen detection and early warning systems. Microfluidic systems that allow the manipulation of cells, bacteria, and a large number of exceedingly small fluid samples could be used to carry out massive drug discovery trials quickly and with infinitesimal amounts of precious sample materials. There is a clear and large market for miniaturized implantable sense-and-inject drug delivery systems such as glucose monitoring and insulin injection for type I diabetic patients [4].

\subsection{Current Status and Solutions: EWOD Modeling}

Modeling is required both to quantify EWOD system design tradeoffs and to enable control synthesis. Our modeling effort has been split into two parts: minimum energy modeling to understand the effect of applied electric fields on equilibrium liquid shapes and fluid dynamic modeling to quantify droplet motion, splitting, merging, and mixing.

To first order, surface tension can be modeled by assigning an energy to each interface, this energy is given by a material dependent surface tension coefficient times the area of the interface. The electrical energy stored in the dielectric is given by the integral of the electric field dotted with the resulting dipole moment in the dielectric. There is also energy that is stored in the charging source: this energy is always twice the energy of the dielectric but with opposite sign [30]. The equilibrium liquid shape corresponds to an energy minimum of the total surface tension and electrical energies. In [16] we found the equilibrium shape of a single droplet, on a planar surface, for different liquid and solid materials under the action of an electric field. This result was extended to droplets confined in solid geometries of any shape in [31].

Fluid dynamic simulations are necessary to quantify droplet motion. Here we model the bulk liquid via the Navier Stokes equations. The continuum assumptions behind the NS equations are still valid because the EWOD micro-meter device length scales are far greater than the mean free path of both air and water molecules. However, the small length scales imply that the convective momentum terms are negligible and so these terms are discarded. The motion of the air around the liquid droplets is also neglected.

We track the location of the moving liquid/gas interfaces by the level set method [32, 33] which implicitly defines the liquid boundaries as the zero level set of a scalar function that is convected by the liquid velocity field. The tracking occurs in two spatial dimensions only: the threedimensional interface is recreated by assuming a circular cross section from top to bottom. This approximation is accurate when the liquid/gas interface spans the vertical distance between the floor and ceiling, it is not accurate at liquid pinch regions where the liquid neck separates away from the top and bottom surfaces. The liquid/gas boundary conditions depend on curvature which involves second order spatial derivatives of the level set. To limit the buildup of numerical noise, we filter the level set data before the curvature computation and then post-filter the 
curvature data at each time step. To ensure mass conservation we correct the level set by a small constant value at each time.

The most crucial part of the model is the definition of the boundary conditions at the liquid/gas interface. To match experimental data, we include both the obvious boundary forces due to surface tension effects based on the curvature of the liquid/gas front and the forces due to the EWOD actuation; as well as less obvious boundary forces due to the loss mechanisms of contact angle saturation, stiction (contact line pinning), and contact angle hysteresis. This resulted in a good match between theory and experiment as shown in Figure 4. A detailed description of our fluid model can be found in [34].

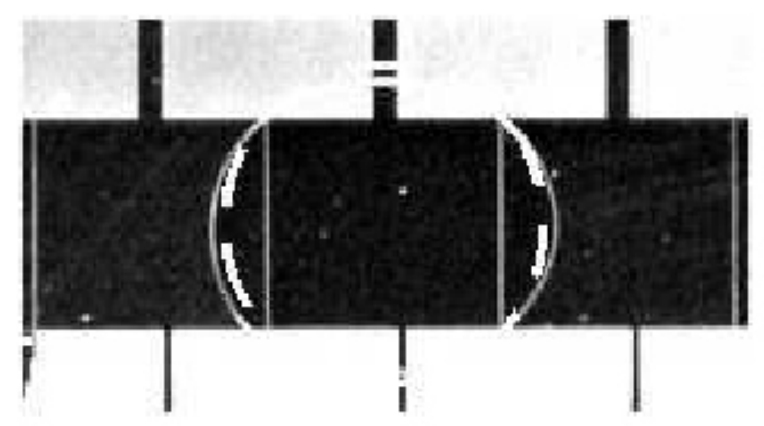

(a) Time: $0 \mathrm{~ms}$.

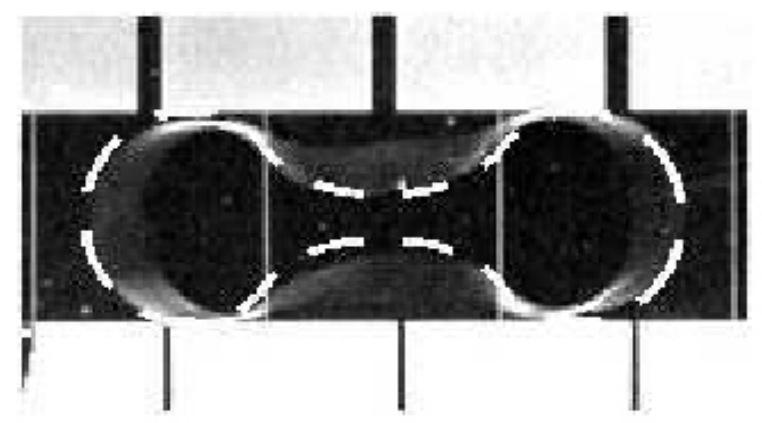

(c) Time: $66.67 \mathrm{~ms}$.

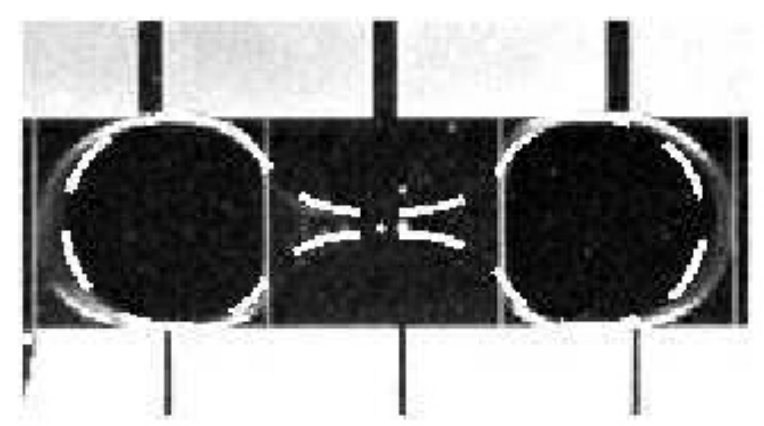

(e) Time: $133.33 \mathrm{~ms}$.

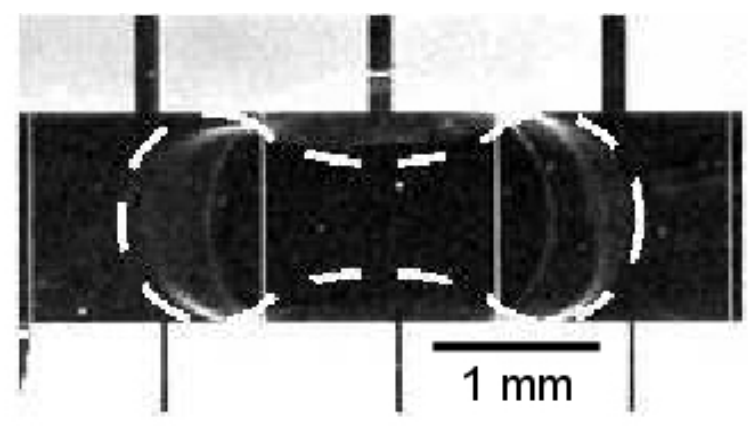

(b) Time: $33.33 \mathrm{~ms}$.

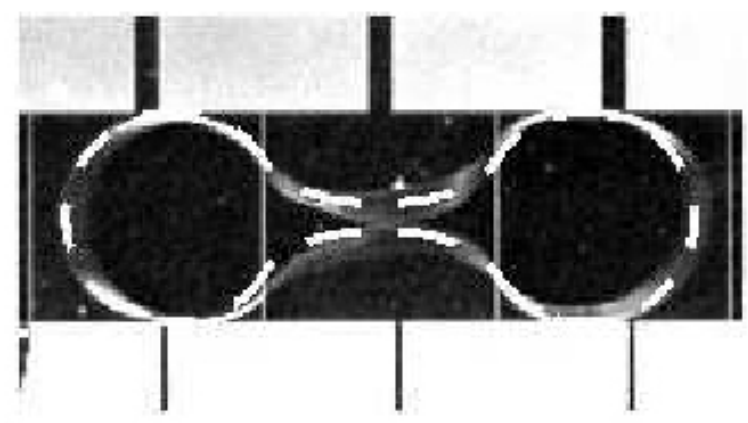

(d) Time: $100.0 \mathrm{~ms}$.

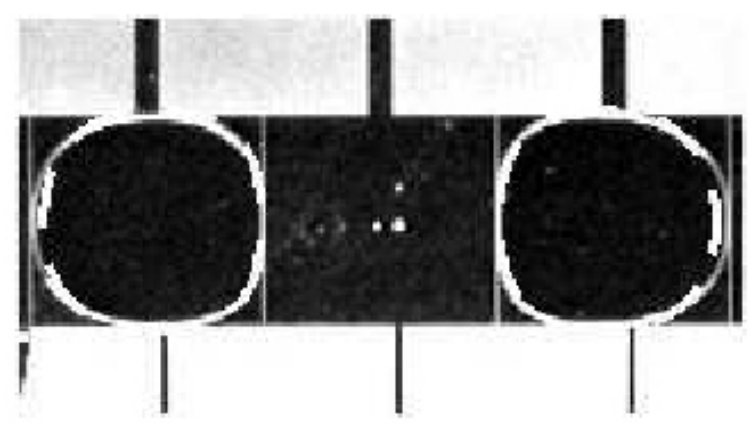

(f) Time: $166.67 \mathrm{~ms}$.

Figure 4: Droplet splitting in the UCLA EWOD devices (looking from above through the top transparent [light yellow] electrode shown in Figure 3). The electrodes on either side of the drop of water are turned on. 
This effectively decreases the hydrophobicity of the solid on either side and pulls the droplet apart. The thin vertical white lines show the boundaries between the underlying electrodes. The blurred white curve is the observed liquid/gas front (the droplet splits apart in two tenth of a second and the $30 \mathrm{~Hz}$ camera cannot fully capture the motion). The thick dashed white line is an overlaid simulation taken from our results in [34].

\subsection{Current Status and Solutions: EWOD Control}

The overall goal is precise and robust control of liquid motion inside the EWOD system. Specific tasks include precision splitting of droplets, control for minimal power and time, trajectory generation for optimal mixing, control of bio-particles inside the droplets, and control for environment and system uncertainty. The current EWOD system is controlled by simple open loop schemes. To move a droplet left an electrode to the left of the droplet is activated; to split a droplet electrodes are activated on either side of the droplet. For now, the desired sequence of droplet motions is pre-programmed. More sophisticated closed-loop control is required for one of three reasons: to manage uncertainty (grains of dust can interrupt EWOD operation), to improve performance (faster mixing), or to enable tasks that are not possible without sophisticated control (precision droplet splitting).

Any EWOD feedback control system will have both a sensing and a control algorithms component. The sensing task is addressed by implementing a vision detection algorithm to determine the shape of the liquid/gas interface in real time. The algorithm proceeds as follows. First we take a background "template" image of the EWOD device when there is no liquid. Then, at each time step, we subtract the image of the device without liquid away from the current camera image of the device with liquid. This isolates all the pixels that correspond to the visible liquid/gas interface. We then perform Gaussian smoothing to remove image noise and apply standard edge detection techniques [35] to find the location of the interface.
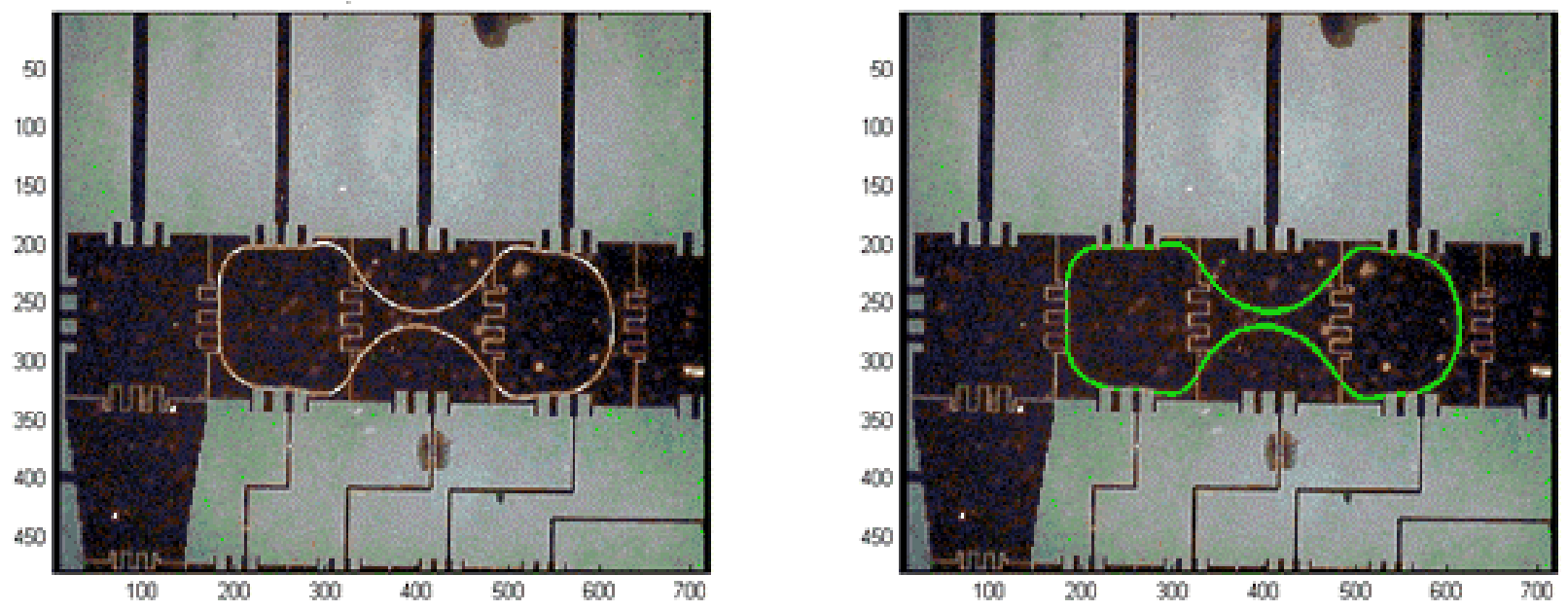

Figure 5: The real time vision based liquid/gas interface detection algorithm. The left side shows a raw camera image of the EWOD device with a liquid drop that is just about to split. The curve on the right side shows the shape of the liquid/gas interface that has been identified by the vision algorithm. 
We have not yet addressed the design of feedback controllers for EWOD uncertainty. For example, we have not yet designed actuation algorithms to correct for liquid interfaces that have been disturbed or pinned by grains of dust or other contaminants. However, we have addressed two sub-tasks and have verified the solutions via our simulations.

\subsubsection{Steering Material Points Inside and on the Boundary of EWOD Droplets}

Techniques from control theory can be used to steer material points inside and on the boundary of the EWOD droplets. This is useful because it allows the steering of biological particles, such as cells or bacteria, to specific sensing locations and it allows steering of material points on the liquid/gas boundary. The EWOD particle steering control task is shown in Figure 6: given the current liquid shape (which in the case of Figure 6 corresponds to the shape immediately after a small circular drop and a larger elliptical drop have merged), and the position of four particles (in Figure 6 the particles are located at the roots of the arrows), find the electrode actuation voltages so that all the particles are steered in the direction of the arrows.

The EWOD actuation voltages determine the pressure on the liquid/gas boundary. At low Reynolds number, the pressure inside the droplet is described by Laplace's equation $\nabla^{2} P(\Omega)=0$ which is linear. Hence there is a linear mapping between the applied voltages and the achieved pressure gradient field (the forces) at the 4 particles. If there are $n$ particles to be controlled and the liquid/gas interface overlaps $m$ electrodes, then there are $m$ actuation variables available to control $2 n$ force variables (each particle has an $x$ and a $y$ degree of freedom). The problem is over constrained if $m>2 n$ (as is the case in Figure 6) and is under constrained otherwise.

To find the best possible applied voltages we solve a least squares problem that minimizes the error between the achieved forces and the desired forces. At the next instant in time, the liquid shape changes, there is a new linear mapping between the applied voltages and the forces on the particle, and we then solve this new least squares problem to keep the particles moving along their desired trajectories. We have experimented with different liquid geometries and particle placements and have found that there is a large degree of controllability: if $m>2 n$ then it is usually possible to steer all the particles along the desired directions. It is not possible to steer the particles when there are too many particles or when the distance between the particles is small compared to the distance to the liquid/gas interface or the distance between the actuators. This heuristic observation is based on the cases we have done, we have not yet characterized the degree of controllability in a more rigorous fashion. Our control scheme has been demonstrated in simulation, as shown in Figure 6 and Figure 7. Validation experiments are now being planned at UCLA. 

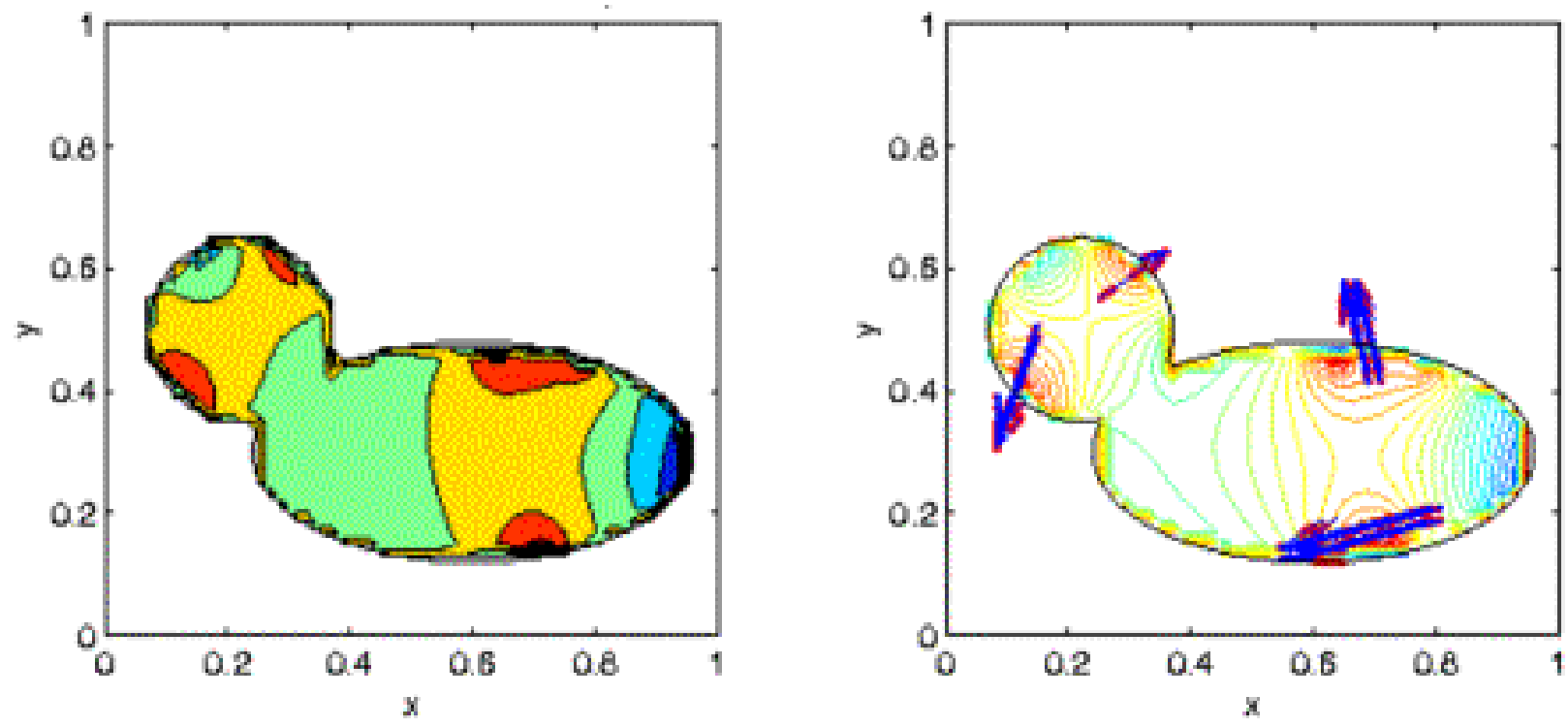

Figure 6: Control of forces at key internal or external points within an arbitrarily shaped droplet. The right figure shows a droplet with 4 embedded particles (at the root of the arrows). The underlying grid of 20 by 20 electrodes is not shown. The question is: what voltages should be applied to create the desired (blue) forces at each of these particles? Since, for any liquid shape, there is a linear mapping between the pressure on the boundary, which is set by the electrode actuation, and the forces on the particles, to find the actuation voltages we solve a least squares problem that minimizes the error between the achieved forces and the desired forces. The resulting pressure field is shown on the left. Contours of that field are shown on the right, along with the achieved forces (red arrows). The match between the blue and red arrows is almost perfect.

We now show how the ideas of Figure 6 can be used to steer a single particle inside an EWOD droplet (the steering is demonstrated using the simulation of [34]). As shown in [34], under assumptions relevant to the EWOD system, there is an ordinary linear differential equation that describes the particle accelerations in terms of the pressure gradients at the particles

$$
\left[\begin{array}{l}
\tau \ddot{x}+\dot{x} \\
\tau \ddot{y}+\dot{y}
\end{array}\right]=-\gamma\left[\begin{array}{l}
\partial P(x, y) / \partial x \\
\partial P(x, y) / \partial y
\end{array}\right] .
$$

Here $\tau$ is a time constant and $\gamma$ is the non-dimensional parameter defined by the ratio of pressure to viscous forces. An analytic solution to equation (1) can be derived to give a relation between the future position of the particle and the current value of the pressure gradient at the particle location. Since we know the current particle velocity and the location desired at the next time step, we can find the desired particle acceleration and can therefore compute the pressure gradient $\nabla \mathrm{P}(\mathrm{x}, \mathrm{y})$ that will achieve the desired particle motion. (For single and multiple particles we solve the least squares problem of Figure 6 to best match the actual pressure gradient to the desired pressure gradient at all particle locations.) Iterating the above procedure at each time step of our simulation, we can make the particle (or particles) follow interesting paths. Figure 7 shows simulation results for steering a single particle around a circular trajectory inside an EWOD droplet. 



Figure 7: EWOD particle control along a circular trajectory. The four figures above (starting at top left) are snapshots from our simulation that show a particle floating inside a moving droplet. The particle is denoted by an asterisk with a short line indicating the current desired velocity. The blue circle denotes the desired trajectory path of the particle; the black curve denotes the liquid-gas interface of the droplet. The dotted lines denote the edges of individual electrodes. As the simulation progresses, the voltages at the electrodes change, thereby changing the pressure boundary condition, so that the droplet moves in a way that keeps the particle traveling in a counter-clockwise direction along the trajectory path. Actuation is achieved using a small (realistic) number of electrode actuators. 


\subsubsection{Control for Precise Droplet Splitting}

Based on the particle steering method above, we have also addressed the task of precision droplet splitting. At present the EWOD devices function in open loop: this means that a droplet can be split into 50\% / 50\% with an approximate variation of $\pm 20 \%$. To split a droplet into $82 \%$ / $18 \%$ by volume, we plan to steer two material points on the boundary to meet along a trajectory that bisects the droplet in the correct ratio. This is a simple way of using the techniques of Figure 6 to achieve precise droplet splitting. The instantaneous droplet shape is available via our real time image processing algorithms as shown in Figure 5.

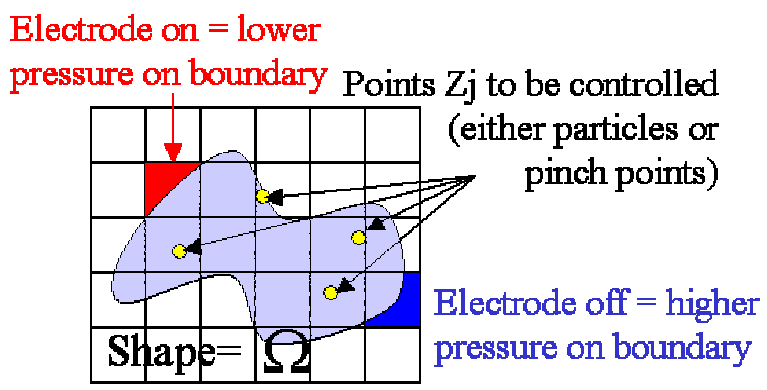

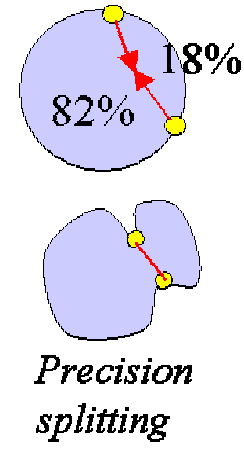

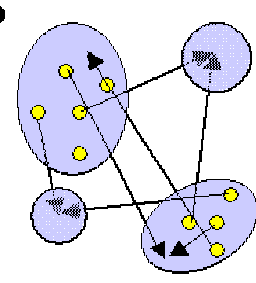

Arbitrary split/ join

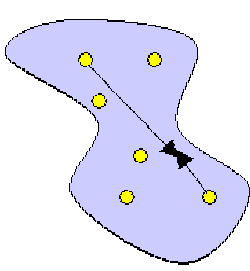

Controlled collisions

Figure 8: Framework for precision control of droplet splitting and embedded particle control. Turning on an electrode lowers the pressure on the liquid/gas boundary locally and creates pressure gradient forces inside the liquid droplet. For control of splitting we choose two material points on the liquid/gas boundary, and we then make those two points collide along a set path that bisects the droplet in the right ratio - this enforces accurate splitting. The control of the material points is achieved as in Figure 6 . The same basic technique can also be used to enforce particle to particle collisions and to sort particles between droplets.

\section{Micro-Flow Control for Particle Steering}

Our goal here is to create microfluidic devices and control algorithms that will independently steer many particles at once by creating underlying fluid flows that will carry all particles along their desired trajectories. The basic idea is shown in Figure 9: by actuating the vertical electrodes it is possible to create complex and time-varying planar flow fields that can carry many particles at once along independent trajectories. The flow fields are created by electroosmotic forces [36, 37] that are described in Section 4.1. In order to carry out the particle steering we combine this device in closed loop with a particle vision sensing system and a feedback controller.

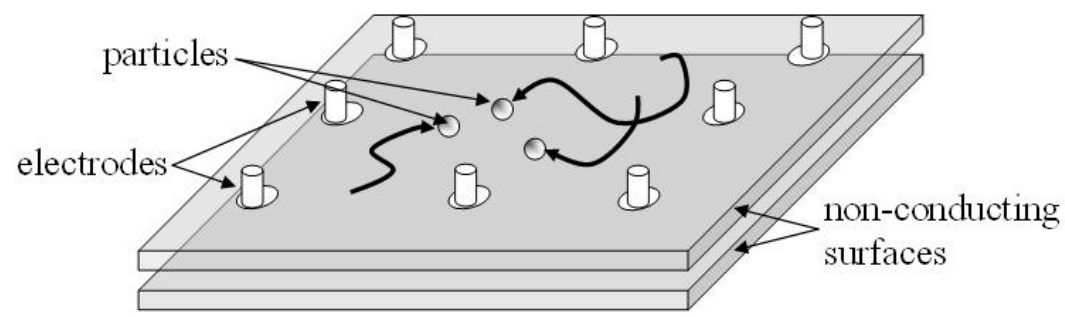

Figure 9: Schematic of the particle steering microfluidic system. The vertical inserted electrodes create complex time-varying planar fluid flows that can be used to steer many particles at once. Each particle has two ( $x$ and $y$ ) degrees of freedom, so $n$ particles require at least $m=2 n+1$ electrodes for steering control (one of the electrodes is grounded so there is only $m-1$ degrees of actuator freedom). In addition, there are situations 
that approach singularity and that cannot be controlled: it is not possible to create flows that violate conservation of mass, and it is not possible to steer nearby particles using electrodes that are far away.

To achieve our particle steering results we require the advantages of both microfluidic systems and feedback control. The particles will be disturbed away from their nominal positions by thermal (Brownian) noise, spurious pressure forces (as created by surface tension and electroloysis at the electrode wells), and electrode actuation errors. These small deviations will be amplified by the spatially complex, time varying flow patterns and the true path of the particles will diverge away from the desired paths. Feedback control is required to continually bring the particles back to their desired paths. Interestingly, in addition to feedback, the physics of fluid dynamics that occurs on the micro scale is also required. On the macro scale, fluid momentum effects create extremely complex fluid flows. It is not mathematically practical to invert the Navier Stokes equations in order to find the actuator inputs that will create the right flows to carry multiple particles in desired directions. By comparison, micro-flows are more predictable. Specifically, the low-Reynolds limit of the Navier Stokes equations gives a linear set of equations that can be effectively inverted: we can determine the necessary input voltages that will steer many particles at once in the desired directions.

Figure 10 shows our experimental results for steering a single yeast cell. There are a number of potential applications for this flow control particle steering technology. It will allow the steering of cells, bacteria, and other microscopic objects to sensor locations, into drug packets, and into each other. We are collaborating with Cristian Ionescu-Zanetti and Luke Lee at Berkeley [3] to steer cells for improved microfluidic patch clamping. (Patch clamping is a technique used to measure the electrical resistance of cell membranes which provide information on the behavior of membrane ion channels whose properties are central to the nervous system and often act as targets for drugs.) The particle steering method can also allow combinatoric biological testing: for a microfluidic system containing many types of cells, bacteria, and drug packets, we wish to steer different cells into different bacteria and then, based on the observed results, steer the outcomes to appropriate drug packets.

The particle steering capabilities of our system are similar to the ones that can be achieved by laser tweezers [38-42]. Compared to laser tweezers, our approach has the advantage that the entire system is miniaturizable (the microfluidics, optics, miniaturized camera, and computer processing can be reduced to a hand held size with existing technology or into the size of match box using CMOS on-chip cameras [43]), and we can steer particles that laser tweezers cannot capture (lasers trap transparent dielectric particle with a refractive index greater than the surrounding medium [44] whereas we can steer any visible object). However, our approach does not have the same tens of nanometer positioning accuracy and three-dimensional control that is available with laser tweezers [42].

\subsection{Current Status and Solutions: Modeling of Particle Steering}

The microfluidic device of Figure 10 uses electroosmotic actuation [36, 37] to create fluid flow. Electroosmotic actuation is routine on the micro-scale. The electric field actuates a thin layer of charges that form naturally at the liquid/solid interface (the amount and sign of the charges 
depends on the chemistry of the materials). This thin moving layer of charges then drags the fluid by viscous forces. This means that the micro-flow will follow the electric field that is active at the floor and ceiling of the device. In our case, the electric field is uniform in the vertical direction but it has complex patterns in the horizontal $(\mathrm{x}, \mathrm{y})$ plane. The resulting micro-flow will exhibit these same complex horizontal patterns (see [45] for details)

(2) $\vec{V}(x, y, z)=(\varepsilon \xi / \eta) \vec{E}(x, y)=-(\varepsilon \xi / \eta) \nabla \phi(x, y)$.

Here $\vec{V}$ is the flow velocity, $\vec{E}$ is the electric field which is uniform in the vertical direction, $\phi$ is the electric potential as created by the actuators of Figure 9, Figure 10, or Figure 11, $\varepsilon$ is the permittivity of the liquid, $\eta$ is its density, and $\xi$ is the zeta potential (essentially the voltage) at the liquid/solid interface [36, 37].

The floating neutral particles are simply carried along by the flow. Thus the particle positions are convected by $\dot{\vec{P}}_{j}=\vec{V}\left(\vec{P}_{j}\right)+\vec{w}$ where $\vec{w}$ is Brownian noise and $\vec{P}$ is the vector of particle $\mathrm{x}$ and $\mathrm{y}$ positions. The electric potential is described by Laplaces equation $\nabla^{2} \phi=0$ with Dirichlet boundary conditions at the electrode boundaries $\phi\left(\partial D_{j}\right)=u_{j}$ where $\partial D_{j}$ denotes the liquid/ electrode interface location and $u_{j}$ is the $\mathrm{j}^{\text {th }}$ applied voltage. Insulating Neumann conditions hold at all other surfaces. The solution of Laplaces equation is linear in the applied voltages so

$$
\dot{\vec{P}}=\vec{V}(\vec{P}, z)+\vec{w}=-(\varepsilon \xi / \eta) \nabla \phi(\vec{P})+\vec{w}=-(\varepsilon \xi / \eta) \sum_{j=1}^{n} \nabla \Phi_{j}(\vec{P}) u_{j}+\vec{w} \triangleq A(\vec{P}) \vec{u}+\vec{w}
$$

where $\Phi_{j}$ is the solution to Laplaces equation when electrode $\mathrm{j}$ has a unit applied voltage and all other electrodes are at zero voltage, and $\vec{u}$ is the time-varying vector of applied voltages. Note that the velocities of the particles depends on where they are with respect to the electric potential $\phi(x, y)$. For the same set of voltages, two different particles in two different locations will execute different motions. In summary, the equations to be controlled are linear in the control and nonlinear in the particle positions: $\dot{\vec{P}}=A(\vec{P}) \vec{u}+\vec{w}$.

\subsection{Current Status and Solutions: Control of Particle Steering}

We have demonstrated steering of one particle experimentally [46] and the steering of many particles at once in simulations that include realistic sources of errors and noise [47]. Recently, we further demonstrated the steering of two particles at once experimentally and we are now fabricating devices for steering five to seven particles.

Experimental results for single particle steering in a microfluidic cross-channel are shown in Figure 10. The microfluidic device was fabricated by molding PDMS to an SU8 template and then bonding the PDMS to a glass slide. Device geometry consists of a cross-channel actuated by four electrodes. The two channels are $2 \mathrm{~cm}$ long, 50 micrometers wide, and 11 micrometers deep. The liquid is a pH 9.3 buffer solution composed of distilled water with 5 molar concentration of Sodium Bicarbonate. Here the zeta potential, which measures the strength of the electroosmotic effect, was measured to be 0.145 milli-volts, this means that the maximum applied voltage of 30 volts creates a fluid velocity of $3.48 \mu \mathrm{m} / \mathrm{s}$. 
The optical system is composed of a Vision-Component 2038 DSP camera and an Axiostar microscope. The real time position of the yeast cell is determined as follows: a previously taken background device image is subtracted from each incoming pixilated camera image, this separates the cell pixels from the background view; the resulting image is now filtered and thresholded; and the center of mass of each blob in the image gives the current position of the yeast cell or cells.

The control algorithm now commands an actuation to move the yeast cell from its current position to the desired position on the 'UMD' path. Each electrode pair corrects for particle position along its own axis independently of the other electrode pair. If the particle is more than five pixels North from its current desired North/South elevation then the North electrode applies plus thirty volts and the South electrode applies minus thirty volts. Once the particle is within five camera pixels a lower twelve volt actuation is used. The East/West electrodes work the same way. This algorithm is sufficient to keep the yeast cell within one pixel of its desired trajectory. Under the microscope magnification, one pixel corresponds to a resolution of one micron and yields the single micron tracking accuracy shown in Figure 10. The above single particle control algorithm is simpler than the one used to steer many particles in Figure 11.

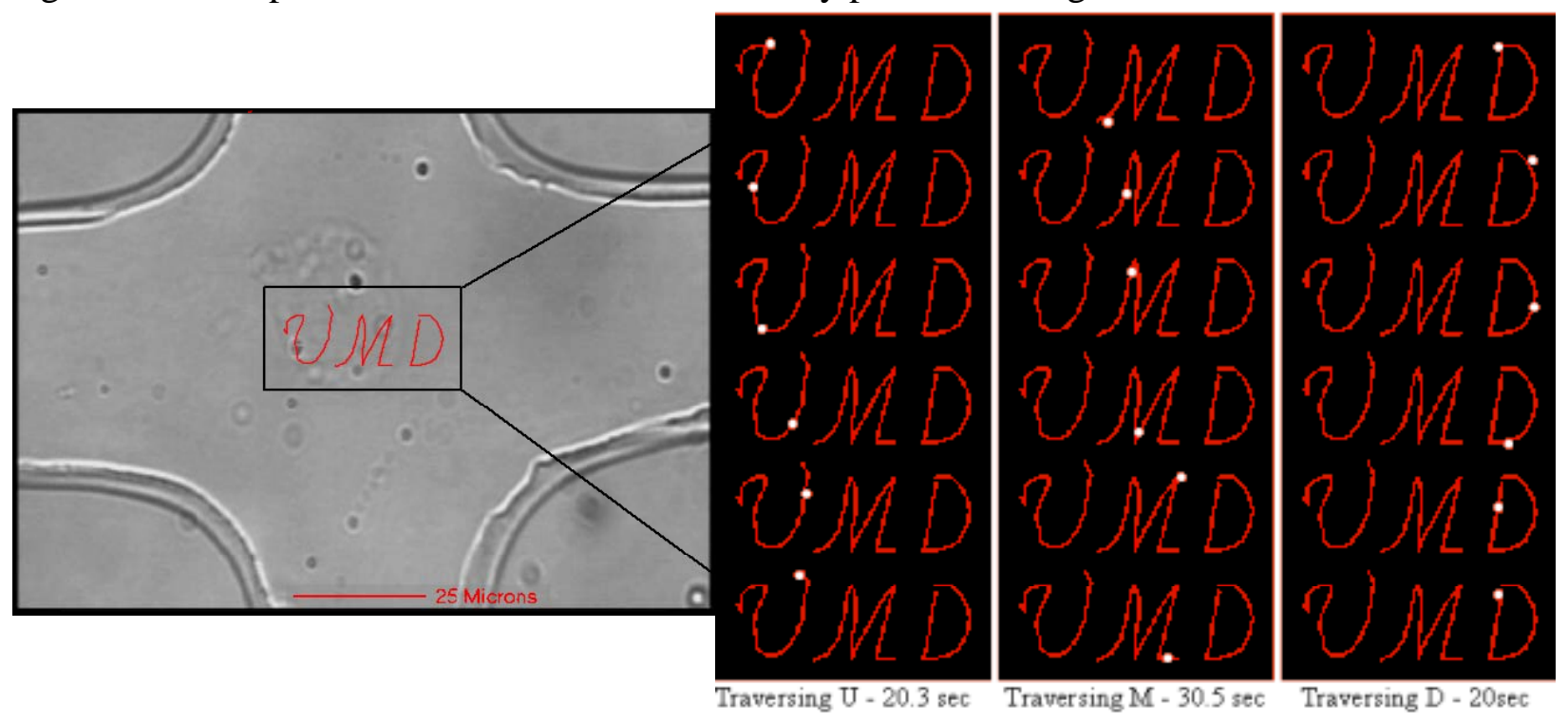

Figure 10: A yeast cell steering experiment. Left: Photograph of the microfluidic devices with the cursive 'UMD' path super-imposed on the image. The four control electrodes are located outside figure frame. Right: The actual path of the yeast cell (white dot) in the feedback control experiment. (Figure taken from [46]). The yeast cell is being steered to an accuracy of one micron. Any other cell that is small enough to act as a particle in the flow could also be steered in this way: the type of cell is not important from a flow control perspective.

Simulated results for multiple particle steering are shown in Figure 11. Here twenty electrodes are used to create complex planar flow patterns that carry all particles along their desired paths. We have been able to demonstrate independent steering of up to ten particles in simulations that include Brownian noise and small sensor and actuator errors. Recently, we have also shown steering of two particles at once experimentally, and we are now fabricating devices that will enable steering of five to seven particles. 



Figure 11: Steering of multiple particles by micro flow control. The view is from above; large gray circles are the actuating electrodes; the small black circles are the particles to be controlled; the color represents the instantaneous voltage field $\varphi(x, y)$ : black/red corresponds to a high voltage, white/yellow corresponds to a low voltage; and the vectors are the resulting fluid velocities which are along the electric field due to electroosmotic and viscous forces. The particles execute the motion shown by the black curves. The two left frames correspond to a simulation according to equation (3) with no noise $(\vec{w}=0)$. In this case, the control is simply open loop as described in the text. The right frame shows a simulation that includes Brownian noise $(\vec{w} \neq 0)$ with a closed loop feedback linearization control scheme. Nineteen SVD modes were used and these SVD modes were discretized over a 100 by 100 grid. Here the thick green curves are the desired trajectories and the thin black curves are the actual particle paths - they overlay almost perfectly.

We split the multi particle steering control into two tasks: open loop control for path generation and closed loop feedback control to correct for noise and sensing/actuation errors. The open loop control is based on a least squares inversion of equation (3). Specifically, the velocity of the particles is given by $\dot{\vec{p}}=A(\vec{p}) \vec{u}$. Our first task is to find the set of voltages $\vec{u}(t)$ that will generate a set of velocities $\vec{v}_{D}(t)=\dot{\vec{p}}_{D}(t)$ along the desired particle paths $\vec{p}_{D}(t)$. The least squares solution $\vec{u}(t)=\left[A^{T}(\vec{p}(t)) A(\vec{p}(t))\right]^{-1} A^{T}(\vec{p}(t)) \vec{v}_{D}(t)$ finds the set of open loop control voltages $\vec{u}(t)$ that will minimize the 2-norm error between the actual and the desired particle velocities $\left\|\dot{\vec{p}}(t)-\vec{v}_{D}(t)\right\|_{2}$. However, as stated, the least squares problem is ill-conditioned: even a $2 \%$ change in particle positions or velocities will lead to $200 \%$ changes in control voltages.

We use singular value decomposition (SVD) modes to condition the least squares problem. The linear mapping from the control voltages to the flow velocity everywhere in the domain is discretized and we decompose the resulting matrix into its SVD modes. The first mode corresponds to the highest flow velocities for the smallest applied voltages, the last mode is the least effective mode with the smallest, localized flow velocities for the highest voltages - it is these higher modes that create the ill-conditioning. By projecting the least squares problem onto the first $k$ modes, we arrive at a well-conditioned least squares control algorithm. This algorithm was used to generate the open loop results of Figure 11. The number of particles that we can steer at once is fixed by our actuator and sensor accuracy: as we attempt to steer more particles, we have to include more SVD modes, and this amplifies the sensor errors into larger control voltage fluctuations. For realistic system settings of noise and sensing/actuation errors, we can steer about ten particles at once in simulations. 
Feedback linearization [48] is used to correct for particle deviations away from the nominal trajectories due to Brownian noise. Let $\vec{P}(t), \vec{U}(t)$ be the nominal open loop particle positions and control voltages, and let $\vec{p}(t)=\vec{P}(t)+\Delta \vec{p}(t), \vec{u}(t)=\vec{U}(t)+\Delta \vec{u}(t)$ be the actual particle positions and the actuator voltages applied by the closed loop control system. By using the feedback law $\Delta \vec{u}=\left[A^{\mp}(z)\right](-a \Delta \vec{p}+[A(\vec{P})] \vec{U})-\vec{U}$ where $A^{\mp}=\left(A^{T} A\right)^{-1} A^{T}$ is the pseudo-inverse of $A$, projected onto the first $k$ SVD modes as before, and $a$ is a scalar quantity. This feedback linearization scheme creates globally stable linear dynamics in the particle deviation $\Delta \dot{\vec{p}}=-a \Delta \vec{p}+\vec{w}$ from the nominal. Closed loop results are shown on the right of Figure 11.

The multiparticle steering problem is solvable because, for any instantaneous placement of the particles, there is a linear mapping between the applied actuator voltages and the particle velocities: the low Reynolds limit of the Navier Stokes equations yields linear Stokes fluid flow equations. This linear mapping can be inverted, after pre-conditioning by SVD, to find the actuator inputs that will move all the particles in the desired directions at each time. Here the SVD fluid modes are pre-computed but the least squares problem, which depends on the particle positions, is solved at every time step. The SVD modes have the additional advantage that they show which fluid modes require low actuation energies (are easy to achieve) and which modes require high control energy. Not surprisingly it is not possible to create flow fields that violate conservation of mass, and it is difficult to independently control particles that are very close to each other. Besides this, we have not found any particle configurations that are singular and cannot be controlled. The magnitude of the disturbance noise $w$, which for the non-dimensional formulation is the ratio between the thermal and electrical actuation forces, depends on the temperature, particle size, and applied voltage, but is typically very small (less than one percent, see [47]). As a result, the open loop control effort is much greater than the closed loop corrections.

In terms of experimental demonstrations, there are two key practical concerns that must be addressed. Actuation and sensor errors, as well as errors due to other physical effects (such as spurious pressure forces created by surface tension forces and electrolysis at the electrode wells), must be minimized. The dominant device flow modes must be validated experimentally: although the simulations of Section 4.1 are quantitatively correct, they do not account for all the possible variations that occur in the experiments. We are using micro Particle Image Velocimetry ( $\mu$ PIV) techniques, the fluid is seeded with visible particles and tracking of the particles reveals the flow field [49,50], to experimentally characterize the fluid flow in our devices.

Finally, it is interesting to compare the controllability of particles in the EWOD example of Figure 6 with the particle controllability shown above in Figure 11. We have not yet addressed this question in a rigorous manner but we can make the following comparison. When we apply control through actuators at the boundary of a domain, the resulting flow field is smoothed by diffusion, fine scale information is lost, and it is difficult to steer nearby particles in different directions. In the electroosmotic particle steering task there is one level of smoothing between the actuator inputs and the particle velocities: 


$$
\left[\phi\left(\partial \Omega_{k}\right)=u_{k}\right] \rightarrow\left[\nabla^{2} \phi(x, y)=0\right] \rightarrow\left[\dot{p}_{j}=-\nabla \phi\left(p_{j}\right)\right] .
$$

Only one Laplacian smoothing operator is between the control $u$ and the particle velocity $d p / d t$. In the electrowetting case there are two such operators. The two operators come from the low Reynolds limit of the Navier Stokes equation: the first operator deals with the pressure field, and the second Laplacian operator maps from the applied pressure gradient to the fluid velocity:

$$
[P(\partial \Omega)=u] \rightarrow\left[\nabla^{2} P(x, y)=0\right] \rightarrow\left[\nabla^{2} \vec{v}(x, y)=\nabla P\right] \rightarrow\left[\dot{p}_{j}=\vec{v}\left(p_{j}\right)\right] .
$$

Hence there is more particle steering control authority available in the electroosmotic particle steering test-bed than there is in the EWOD systems.

\section{Common Themes and Challenges in Control of Microfluidic Systems}

There is an overlap between the needs found in micro systems and the tools available in control theory. By definition, micro systems have components with micrometer sizes: this means that it is possible to pack numerous actuators and sensors into tiny volumes. (Yet, in most practical microfluidic systems, the number of actuators per particle, cell, DNA chain, or fluid droplet remains small. The point of miniaturization is, usually, to handle many miniature entities: not to address a small number of macro entities with a large number of small actuators.) Nevertheless, the need to coordinate many micro sensors and actuators matches the distributed control capabilities developed by control theorists.

Moreover, micro-systems, especially those that are integrated with centimeter scale human interfaces, have length and time scales that vary over many orders of magnitude. This matches control analysis and design tools that have been created to capture sub-system coupling across disparate length and time scales. Many physical phenomena that are found on the micro scale are poorly understood; plus micro systems that are created at the limits of fabrication resolutions can have significant variations in device geometries; and micro systems typically handle biological materials that can vary significantly from sample to sample. This means that there is a large amount of dynamic, geometric, and parametric uncertainty in micro-systems. The ability to analyze and design for uncertainty is a staple of control theory. Hence there is a clear potential for control theory to make a strong impact in the advancement of micro- and nano-scale science.

We have found that the challenges in control of microfluidic systems are, in chronological order for each new project: identifying interesting applications that may require control; modeling for control; paraphrasing micro control tasks as tractable mathematics problems; experimental validation; and effectively communicating with fabricators, chemists, and biologists. Of these, the first and last challenges are best handled by continual interaction with colleagues and collaborators in other fields. The other three items are discussed below.

The first, and usually central, task in microfluidic modeling is to determine which physical phenomena dominate in a given system. The answer depends on the system being considered, and the development of appropriate numerical methods can only proceed once this question has been resolved. In the electroosmotic system, the dominant phenomenon is the viscous coupling of Stokes flow with the thin Deybe layer that is driven by electrostatic fields. In the EWOD 
system there is a competition between surface tension and solid dielectric polarization energies that create the actuation forces, and these then drive the two-phase Stokes flow. EWOD fluid dynamics is further affected by various loss mechanisms. For example, possible phenomena leading to contact angle saturation include: electrolysis, electrostatic/capillary instabilities, ionization of air in the vicinity of the triple line, charge/ion adsorption from liquid to the solid surface, charge trapping, and electrical losses inside non-ideal dielectric materials. It turns out that, in the UCLA EWOD system, contact angle saturation is caused by a small amount of liquid resistance that progressively deprives the solid dielectric of the electric field it needs to effect a continued shape change [16].Other loss mechanisms, such as contact line pinning and contact angle hysteresis, also had to be understood and included in the boundary conditions of our simulations in order to match experimental data [34].

Paraphrasing micro control tasks as tractable mathematics problems is necessary to provide a link between micro-scale applications and available control techniques. Typically, finding a good way to state the control problem is more challenging than solving the control problem once it has been stated. For example, it is clear that precision droplet splitting under vision sensing is some kind of a feedback control task. By phrasing this question as the steering of two material points by the method of least squares (see Figure 6 and Figure 8) we have provided a link between the practical application needs and a viable mathematical tool. If we had tried to directly control the shape of the entire droplet by using, or extending, current partial differential equation control methodologies, we would have arrived at an extremely complex control algorithm that could not be implemented in real time.

Experimental validation relies on having access to experiments. Micro fabrication technologies are expensive and microscale experiments are delicate and time-consuming (even by the standards of experimental research in general). In principle, a viable strategy for a control theorist is to collaborate with a micro-fabrication laboratory - then the control theorist can focus on control and the micro researchers can focus on fabrication and experiments. In practice, this is a realistic plan so long as the research goals of the control researcher are a sub-set of the research goals of the micro fabrication laboratory. In order to demonstrate particle steering by flow control, a research topic that to the best of our knowledge is not being pursued by other researchers, we had to fabricate our own devices. The benefit is that we have demonstrated, to the micro-electro-mechanical-systems (MEMS) community, a microfluidic capability that is simply not possible without feedback control.

In order to facilitate further collaboration between control scientists and micro/nano researchers, the author initiated and organized the March 2004 NSF workshop on 'Control and System Integration of Micro- and Nano-Scale Systems' [5]. The workshop brought together ninety experts in modeling, control, micro/nano fabrication, and bio-chemical systems for two days to " ... identify research areas, to foster inter-disciplinary collaborations, and to recommend future research directions to NSF that will enable control and system integration on the micro- and nano-length scales". Not surprisingly, the themes and challenges raised at the workshop were similar to the themes and challenges stated above. Specifically, workshop participants outlined the need for micro/nano system integration analysis tools such as reduced order modeling techniques; they identified high-payoff areas for feedback control including fabrication process 
control, control of atomic force microscopy (AFM) probes, and on-chip MEMS and microfluidic control; and they outlined educational and infra-structure needs including the need for co-advised students, cross-disciplinary faculty, and summer workshops that would expose controls faculty to micro- and nano-scale fabrication issues. The final report from the workshop can be found online at the workshop web-site [5].

\section{Conclusion}

There is an overlap between the research needs in micro- and nano-scale systems and the tools provided by feedback control scientists. Micro systems can have large geometric, parametric, and dynamic uncertainties; micro components are now being combined into sophisticated systems, and the internal and external physical behavior of micro devices varies over a wide range of length and time scales. This matches control strength in robust analysis/design, system integration, and modeling across disparate length and time scales. In this paper we discuss two microfluidic systems that can benefit from, and require, feedback control. The first system is the EWOD system developed at UCLA that uses electrically actuated surface tension forces to move, split, merge, and mix liquids on-chip. The second system is a electroosmotic flow control particle steering system developed and experimentally validated in the authors laboratory. Based on these two examples and the results of the NSF workshop on 'Control and System Integration of Micro- and Nano-Scale Systems', we outline some common themes and challenges as they pertain to the control scientist interested in performing research at the intersection of feedback control and miniaturized systems.

\section{References}

1. Northrup, M.A., et al., A Miniature Analytical Instrument for Nucleic Acids Based on Micromachined Silicon Reaction Chambers. Analytical Chemistry, 1998. 70(5): p. 918922.

2. Figeys, D., Adapting Arrays and Lab-on-a-chip Technology for Proteomics. Proteomics, 2002. 2(4): p. 373-382.

3. Seo, J., et al., Integrated multiple patch-clamp array chip via lateral cell trapping junctions. APPLIED PHYSICS LETTERS, 2004. 84(11): p. 1973-1975.

4. Spera, G., Implantable Pumps Improve Drug Deliver, Strengthen Weak Hearts. Medical Devices \& Diagnostic Industry Magazine, 1997.

5. Shapiro, B., Website for the NSF workshop on 'Control and System Integration of Microand Nano-Scale Systems': http://www.isr.umd.edu/CMN-NSFwkshp/. 2004.

6. Gad-el-Hak, M., The Fluid Mechanics of Microdevices -- The Freeman Scholar Lecture. Journal of Fluids Engineering, 1999. 121: p. 5.

7. Beskok, A. Physical Challenges and Simulation of Micro Fluidic Transport. in 39th Aerospace Sciences Meeting \& Exhibit. 2001. Reno, Nevada: AIAA.

8. Karniadakis, G.E. and A. Beskok, Micro Flows: Fundamentals and Simulation. 2001, New York, NY: Springer Verlag.

9. Ho, C. and Y. Tai, Micro-Electro-Mechanical-Systems (MEMS) and Fluid Flows. Annu. Rev. Fluid Mech., 1998. 30: p. 579-612. 
10. Darhuber, A.A., et al., Thermocapillary actuation of liquid flow on chemically patterned surfaces. Physics of Fluids, 2003. 15(5): p. 10150-10153.

11. Chaudhury, M.K. and G.M. Whitesides, How to make water run uphill. Science, 1992. 256(5063): p. 1539-41.

12. He, B. and J. Lee. Dynamic wettability switching by surface roughness effect. in 16th Annual IEEE International Conference on MEMS. 2003. Kyoto, Japan.

13. Lee, J., et al., Electrowetting and electrowetting-on-dielectric for microscale liquid handling. Sensor Actuat. A-Phys, 2002. 95: p. 269.

14. Vallet, M., B. Berge, and L. Vovelle, Electrowetting of water and aqueous solutions on poly(ethyleneterephthalate) insulating films. The European Physical Journal, 1996. 11(B): p. 583-591.

15. Ren, H., et al., Dynamics of electro-wetting droplet transport. Sensors and Actuators BChemical, 2002. 87(1): p. 201-206.

16. Shapiro, B., et al., Equilibrium Behavior of Sessile Drops under Surface Tension, Applied External Fields, and Material Variations. Journal of Applied Physics, 2003. 93(9).

17. Verheijen, H.J.J. and W.J. Prins, Reversible electrowetting and trapping of charge: Model and experiments. Langmuir, 1999. 15: p. 6616-6620.

18. Seyrat, E. and R.A. Hayes, Amorphous fluoropolymers as insulators for reversible lowvoltage electrowetting. Journal of Applied Physics, 2001. 90(3).

19. Hinze, J.O., Turbulence. 2 ed, ed. B.J. Clark. 1975, New York, NY: McGraw-Hill Book Company.

20. Oddy, M.H., J.G. Santiago, and J.C. Mikkelson, Electrokinetic instability micromixing. Analytical Chemistry, 2001. 73(24): p. 5822-32.

21. Stroock, A.D., et al., Chaotic Mixer for Microchannels. Science, 2002. 295.

22. El Moctar, A.O., N. Aubry, and J. Batton, Electro-hydrodynamic micro-fluidic mixer. Lab on a Chip, 2003. 3(4): p. 273-280.

23. Mensing, G.A., et al., An externally driven magnetic microstirrer. Philosophical Transactions of the Royal Society of London Series A-Mathematical Physical and Engineering Sciences, 2004. 362(1818): p. 1059-1068.

24. Fusayo, S., et al., Electrowetting on Dielectrics (EWOD): Reducing Voltage Requirements for Microfluidics. Polymeric Materials: Science \& Engineering, 2001. 85: p. 12-13.

25. Kim, C.J. and J. Lee. Microactuation by Continuous Electrowetting Phenomenon and Silicon Deep RIE Process. in ASME Int. Mechanical Engineering Congress and Exposition. 1998. Anaheim CA.

26. Kim, C.J. and J. Lee. Liquid Micromotor Driven by Continuous Electrowetting. in IEEE Micro Electro Mechanical Systems Workshop. 1998. Heidelberg, Germany.

27. Yang, J., C.W. Li, and M.S. Yang, Lab-on-a-chip (Microfluidics) Technology. Acta Biochimica et Biophysica Sinica, 2002. 34(2): p. 117-123.

28. Verpoorte, E., Microfluidic Chips for Clinical and Forensic Analysis. Electrophoresis, 2002. 23(5): p. 677-712.

29. Kutter, J.P., Current Developments in Electrophoretic and Chromatographic Separation Methods on Microfabricated Devices. Trac-Trends in Analytical Chemistry, 2000. 19(6): p. 352-363. 
30. Feynman, R.P., R.B. Leighton, and M. Sands, The Feynman Lectures on Physics. 1964: Addison-Wesley Publishing Company.

31. Fortner, N. and B. Shapiro, The Equilibrium Shape of Liquids Under Electric Fields. Lab-On-A-Chip, 2005: p. submitted.

32. Sethian, S.A., Level Set Methods \& Fast Marching Methods. 2 ed. 1999: Cambridge University Press.

33. $\quad$ Osher, S. and R. Fedkiw, Level Set Methods and Dynamic Implicit Surfaces. 2003: Springer-Verlag New York.

34. Walker, S. and B. Shapiro, Modeling the Fluid Dynamics of Electro-Wetting on Dielectric (EWOD). Journal of Micro-Electro-Mechanical Systems, 2004: p. submitted.

35. Davies, E.R., Machine Vision: Theory, Algorithms and Practicalities. 1990: Academic Press.

36. Probstein, R.F., Physicochemical Hydrodynamics: An Introduction. 2 ed. 1994, New York: John Wiley and Sons, Inc.

37. Hiemenz, P.C. and R. Rajagopalan, Principles of Colloid and Surface Chemistry. 3 ed. 1997, New York, Basel, Hong Kong: Marcel Dekker, Inc.

38. Chi-Kuang, S., H. Yin-Chieh, and C. Ping Chin, Cell manipulation by use of diamond microparticles as handles of optical tweezers. Journal of the Optical Society of America, B. Optical Physics, 2001. 18(10): p. 1483-1489.

39. Gosse, C. and V. Croquette, Magnetic tweezers: micromanipulation and force measurement at the molecular level. Biophysical Journal, 2002. 82(6).

40. $\quad$ Sheetz, M.P., Laser Tweezers in Cell Biology. Methods in Cell Biology. Vol. 55. 1998: Academic Press.

41. Wang, M.D., et al., Stretching DNA with optical tweezers. Biophysical Journal, 1997. 72(3).

42. Curtis, J.E., B.A. Koss, and D.G. Grier, Dynamic holographic optical tweezers. Optics Communications, 2002. 207(1-6): p. 169-75.

43. Fossum, E.R., CMOS Image Sensors: Electronic Camera-On-A-Chip. IEEE Trans. On Electron Devices, 1997. 44(10): p. 1689-1698.

44. Ashkin, A., History of Optical Trapping and Manipulation of Small-Neutral Particles, Atoms, and Molecules. IEEE Journal on Selected Topics in Quantum Electronics, 2000. 6(6): p. 841-856.

45. Chaudhary, S. and B. Shapiro, Arbitrary Steering of Multiple Particles at Once in an Electroosmotically Driven Micro Fluidic System. IEEE Trans. on Control Systems Technologies, 2004: p. submitted.

46. Armani, M., et al. Using Feedback Control and Micro-Fluidics to Steer Individual Particles. in 18th IEEE International Conference on Micro Electro Mechanical Systems. 2005. Miami, Florida.

47. Chaudhary, S. and B. Shapiro, Arbitrary Steering of Multiple Particles at Once in an Electroosmotically Driven Microfluidic System. IEEE Transactions on Control Systems Technology, 2005.

48. Khalil, H.K., Nonlinear Systems. 2002: Prentice Hall.

49. Santiago, J.G., et al., A particle image velocimetry system for microfluidics. Experiments in Fluids, 1998. 25(4): p. 316-319. 
50. Wereley, S.T. and C.D. Meinhart, Micron-Resolution Particle Image Velocimetry. NanoScale Diagnostic Techniques, ed. K.S. Breuer. 2004, New York: Springer-Verlag. 